\title{
PRODUÇÃO DE MATÉRIA SECA, CRESCIMENTO RADICULAR E ABSORÇÃO DE CÁlCIO, FÓSFORO E ALUMÍNIO POR Coffea canephora E Coffea arabica SOB INFLUÊNCIA DA ATIVIDADE DO ALUMÍNIO EM SOLUÇÃO(1)
}

\author{
Edson Marcio Mattiello( ${ }^{(2)}$, Marcos Gervasio Pereira ${ }^{(3)}$, Everaldo \\ Zonta $^{(4)}$, Jocimar Mauri ${ }^{(5)}$, José Dioenis Matiello ${ }^{(6)}$, Paulo Geovane \\ Meireles $^{(7)} \&$ Ivo Ribeiro da Silva ${ }^{(8)}$
}

\begin{abstract}
RESUMO
Este trabalho teve como objetivo avaliar a produção de matéria seca, o crescimento radicular e a absorção e distribuição do $\mathrm{Ca}, \mathrm{P}$ e $\mathrm{Al}$ nas folhas, no caule e nas raízes de dois clones de café conilon (Coffea canephora) (Mtl 25 e Mtl 27) e de uma variedade de café Catuaí Amarelo (Coffea arabica), cultivados em solução nutritiva com atividade crescente de $\mathrm{Al}^{3+}$. As plantas foram cultivadas em vasos com capacidade para $5 \mathrm{~L}$, contendo solução nutritiva de Hoagland \& Arnon, modificada. Após oito dias de adaptação, as plantas foram submetidas a concentrações de Al de $0,500,1.000$ e $2.000 \mu \mathrm{mol} \mathrm{L}^{-1}$, que corresponderam a atividades de $\mathrm{Al}^{3+}$ em solução, estimadas pelo software GEOCHEM, de 20,68, 50,59, 132,9 e 330,4 $\mu \mathrm{mol} \mathrm{L}{ }^{-1}$, respectivamente. Foram determinados os teores de Ca, $\mathrm{Al} \mathrm{e}$ $P$ na planta. $O$ sistema radicular foi separado, para determinação da área e do comprimento. A variedade Catuaí Amarelo (Coffea arabica) apresentou-se menos sensível ao $\mathrm{Al}^{3+}$, quando comparada aos clones de conilon (Coffea canephora). O clone de conilon $\mathrm{Mtl} 25$ foi menos sensível ao $\mathrm{Al}^{3+}$ em relação ao $\mathrm{Mtl}$ 27. $\mathrm{O}$ aumento da atividade de $\mathrm{Al}^{3+}$ promoveu redução nos teores de $\mathrm{P}$ e Ca nas folhas e raízes do
\end{abstract}

\footnotetext{
(1) Recebido para publicação em novembro de 2006 e aprovado em outubro de 2007.

(2) Doutorando do Departamento de Solos, Universidade Federal de Viçosa - UFV. CEP 36571-000 Viçosa (MG). E-mail: mattielloem@yahoo.com.br

(3) Professor Associado do Departamento de Solos, Universidade Federal Rural do Rio de Janeiro - UFRRJ. BR 465, km 7, CEP 23890-000 Seropédica (RJ). Bolsista do CNPq. E-mail: gervasio@ufrrj.br

(4) Professor Adjunto do Departamento de Solos, UFRRJ. E-mail: ezonta@ufrrj.br

(5) Mestrando do Departamento de Solos, Universidade Federal de Viçosa - UFV. CEP 36571-000 Viçosa (MG). E-mail: jocimarmauri@yahoo.com.br

(6) Mestrando do Departamento de Biologia Animal, UFV. E-mail: diou2005@yahoo.com.br

(7) Estudante de Agronomia da UFRRJ. E-mail: meirelespg@yahoo.com.br

${ }^{(8)}$ Professor Adjunto do Departamento de Solos, UFV. E-mail: irsilva@solos.ufv.br
} 
cafeeiro, especialmente nos clones Mtl 25 e Mtl 27. O acúmulo de Al no sistema radicular e a restrição do transporte para a parte aérea são importantes fatores na tolerância de plantas ao $\mathrm{Al}^{3+}$.

Termos de indexação: toxidez por alumínio, nutrição mineral, café.

\title{
SUMMARY: DRYMATTER PRODUCTION, ROOT GROWTH AND CALCIUM, PHOSPHORUS AND ALUMINUM ABSORPTION BY Coffea canephora $A N D$ Coffea arabica UNDER INFLUENCE OF ALUMINUM ACTIVITY IN SOLUTION
}

\begin{abstract}
This study had the objective of evaluating the dry matter production, root growth, and the absorption and distribution of $\mathrm{Ca}, \mathrm{P}$ and $\mathrm{Al}$ in the leaves, stem and roots of two Conilon (Coffea canephora) coffee clones (Mtl 25 and Mtl 27) and the coffee variety Catuaí Amarelo (Coffea arabica) grown in nutrient solution with increasing $\mathrm{Al}^{3+}$ activity. The plants were cultivated in $5 \mathrm{~L}$ pots, containing modified Hoagland \& Arnold nutrient solution. After eight days of adaptation, the plants were subjected to Al concentrations of $0,500,1.000$ and $2.000 \mathrm{~mol} \mathrm{~L}^{-1}$, which corresponded to $\mathrm{Al}^{3+}$ activities of 20.68, 50.59, 132.9, and $330.4 \mathrm{mmol} \mathrm{L}^{-1}$, respectively in solution, estimated by the program GEOCHEM. The Ca, $A l$ and $P$ contents were quantified. The root system was separated to estimate area and length. The variety Catuaí Amarelo (Arabic Coffea) was less $\mathrm{Al}^{3+}$ sensitive than the Conilon (Coffea canephora) clones and Conilon Mtl 25 clone was less $\mathrm{Al}^{3+}$ sensitive than Mtl 27. A higher $\mathrm{Al}^{3+}$ activity resulted in a reduction of the $P$ and $\mathrm{Ca}$ content in the coffee leaves and roots, especially in the clones Mtl 25 and Mtl 27. Al accumulation in the root system and the restriction of the transport to the aerial part are important factors in $\mathrm{Al}^{3+}$ tolerance of coffee plants.
\end{abstract}

Index terms: aluminum toxicity, coffee, mineral nutrition.

\section{INTRODUÇÃO}

O Al é o metal mais abundante e o terceiro elemento mais comum na crosta terrestre. Em solos de pH levemente ácido ou neutro, o $\mathrm{Al}$ está, essencialmente, na forma de óxidos ou aluminos-silicatos. Entretanto, quando os solos se tornam mais ácidos, formas fitotóxicas de $\mathrm{Al}$, principalmente $\mathrm{Al}^{3+}$, são liberadas na solução do solo em concentrações que podem afetar o crescimento da planta (Kochian, 1995).

$\mathrm{O} \mathrm{Al}$ acumula-se preferencialmente no sistema radicular das plantas, retardando seu crescimento e desenvolvimento, aumentando o diâmetro das raízes e promovendo a diminuição do número de raízes laterais, as principais responsáveis pela absorção de água e nutrientes. Resultados de pesquisas têm demonstrado que $\mathrm{Al}^{3+}$ no meio de crescimento influencia a absorção de elementos essenciais, como P, Ca e Mg (López-Bucio et al., 2000). Pavan \& Bingham (1982) observaram redução na absorção de $\mathrm{Ca}, \mathrm{Mg}$ e $\mathrm{P}$ e aumento na absorção de $\mathrm{K}$ em cafeeiros cultivados em solução nutritiva, na presença de Al. Esse fato pode se constituir em fator limitante à produtividade do cafeeiro, visto que as principais regiões produtoras de café no Brasil estão localizadas em solos ácidos, caracterizados por baixa saturação por bases e teores elevados de $\mathrm{Al}^{3+}$, suficientes para alterar o crescimento normal de muitas espécies de plantas cultivadas.

$\mathrm{O} \mathrm{Ca}{ }^{2+}$ tem papel central na regulação de muitos processos celulares em plantas, incluindo mitose e citocinese, sinalização celular, gravitropismo, crescimento polar e correntes citoplasmáticas (Huang et al., 1996). A inibição do influxo de $\mathrm{Ca}^{2+}$ para o interior da célula pelo $\mathrm{Al}^{3+}$ é rápida e reversível. Os canais de $\mathrm{Ca}^{2+}$, na membrana plasmática de células da raiz, são muito sensíveis ao Al. Esse bloqueio pode estar envolvido na toxidez por $\mathrm{Al}$ em plantas, pois pode contribuir para o distúrbio da homeostase intracelular do $\mathrm{Ca}^{2+}$ (Silva et al., 2002; Rengel \& Zhang, 2003; Kochian et al., 2004).

Trabalhos pioneiros já ressaltavam a influência do Al na absorção, transporte e utilização do $\mathrm{P}$ (Clarkson, 1967; Bollard, 1983; Foy, 1983). A tolerância ao Al parece estar relacionada com a capacidade de uso eficiente do $\mathrm{P}$, na presença de consideráveis concentrações de $\mathrm{Al}$ nos tecidos (Gaume et al., 2001; Zhu et al., 2002; Zheng et al., 2005). Tal fato seria possível se o $\mathrm{Al}$ fosse complexado com ácidos orgânicos, não interferindo assim no metabolismo do $\mathrm{P}$, conforme sugerido por Cambraia et al. (1983) em 
estudos com cultivar de sorgo tolerante ao Al. Gaume et al. (2001) propuseram que a resistência ao Al pelo milho estava associada à imobilização do $\mathrm{Al}$ pelo $\mathrm{P}$ nos tecidos das raízes.

As plantas apresentam comportamento diferenciado na presença de Al. Essa distinção é observada tanto entre plantas de espécies diferentes como entre variedades de uma mesma espécie (Matsumoto, 2000; Ryan \& Delhaize, 2001; Ma et al., 2002; Kochian et al., 2004; Yang et al., 2005). O problema da toxicidade por $\mathrm{Al}^{3+}$ é particularmente importante em camadas subsuperficiais do solo, pela dificuldade de aplicação profunda da calagem. Uma alternativa para minimizar esse problema, portanto, seria a utilização de espécies e cultivares de plantas tolerantes ao $\mathrm{Al}^{3+}$, elevando-se, dessa forma, a eficiência produtiva das culturas em regiões com limitações por acidez.

Assim, neste trabalho objetivou-se avaliar a produção de matéria seca, o crescimento radicular, a absorção e distribuição do $\mathrm{Ca}, \mathrm{P}$ e $\mathrm{Al}$ nas folhas, no caule e nas raízes de dois clones de café conilon (Coffea canephora) e de uma variedade de café Catuaí Amarelo (Coffea arabica), cultivados em solução nutritiva, com atividade crescente de $\mathrm{Al}^{3+}$.

\section{MATERIAL E MÉTODOS}

O ensaio foi realizado em casa de vegetação, utilizando-se mudas de dois clones (Mtl 25 e Mtl 27) de Coffea canephora, obtidas via propagação vegetativa, $\mathrm{e}$ mudas da variedade Catuaí Amarelo (Coffea arabica), propagadas via sementes. As plantas foram cultivadas em vasos com capacidade para $5 \mathrm{~L}$, contendo solução nutritiva de Hoagland \& Arnon, modificada com as seguintes concentrações: macronutrientes (mmol L-1): N: 8,26, K: 3,0, P: 0,5, Ca: 1,99, Mg: 0,98 e S: 1,03 ; e micronutrientes $\left(\mu \mathrm{mol} \mathrm{L}^{-1}\right)$ : B: $22,9, \mathrm{Cu}$ : 0,14, Fe-EDTA: 446, Mn: 6,29, Zn: 0,65 e Mo: 0,05. Após oito dias de adaptação, as plantas foram submetidas a concentrações de $\mathrm{Al}$ de: $0,500,1.000 \mathrm{e}$ $2.000 \mu \mathrm{mol} \mathrm{L}^{-1}$, que corresponderam a atividades de $\mathrm{Al}^{3+}$ em solução, estimadas pelo software GEOCHEM, de: $20,68,50,59,132,9$ e $330,4 \mu \mathrm{mol} \mathrm{L}^{-1}$, respectivamente. $\mathrm{O} \mathrm{Al}$ foi adicionado na forma de $\mathrm{AlCl}_{3} \cdot 6 \mathrm{H}_{2} \mathrm{O}$. Para maximizar a atividade do $\mathrm{Al}^{3+}$, as soluções foram mantidas a pH 4,0, com ajustes diários. As plantas foram mantidas sob arejamento intermitente, e as soluções nutritivas trocadas semanalmente.

O delineamento estatístico adotado foi o inteiramente casualizado, em esquema fatorial $3 \times 4$, sendo três genótipos de cafeeiro e quatro atividades de $\mathrm{Al}^{3+}$, com três repetições.

Após período de 95 dias, as plantas foram coletadas, separando-se raízes, caules e folhas. O material vegetal foi seco em estufa de circulação forçada de ar, pesado e moído. Após digestão nitroperclórica, foram determinados os teores de $\mathrm{Ca}$ e $\mathrm{Al}$, por meio de espectrofotômetro de emissão óptica com plasma acoplado por indução (Perkin Elmer, modelo Optima $3300 \mathrm{DV}$ ), e de $\mathrm{P}$, por colorimetria. O sistema radicular foi separado, para estimativa da área e do comprimento. Foi feita amostragem de $10 \%$ da massa fresca das raízes, para digitalização. Os eixos radiculares foram dispostos sobre folhas de acetato com auxílio de pinças, evitando-se ao máximo qualquer tipo de sobreposição. As imagens foram obtidas com auxílio de scanner de mesa (HP Scanjet), utilizandose resolução de 100 dpi em fundo preto, de modo a obter o melhor contraste fundo e objeto. Foram quantificados a área e o comprimento radicular por meio de análise digital de imagens, com auxílio do software SIARCS 3.0 (Jorge \& Crestana, 1996).

Os resultados foram submetidos à análise de variância, sendo os diferentes genótipos avaliados por meio de contrastes ortogonais. Considerando as atividades de $\mathrm{Al}^{3+} \mathrm{em}$ solução nutritiva como variável independente, foram ajustadas equações de regressão para cada genótipo utilizado.

\section{RESULTADOS E DISCUSSÃO}

As plantas cultivadas nas maiores atividades de $\mathrm{Al}^{3+}$ apresentaram sintomas característicos de toxidez por $\mathrm{Al}$, como retardamento do crescimento radicular e de parte aérea, redução do número de raízes laterais e maior diâmetro dos eixos radiculares. As raízes também apresentaram maior rigidez e aspecto de "arrepiamento".

A variedade Catuaí Amarelo (Coffea arabica) apresentou maior produção de matéria seca e crescimento radicular, quando comparada ao conilon (Coffea canephora) (Quadro 1). Os clones do café conilon mostraram diferenças significativas na produção de matéria seca da parte aérea e da raiz, com o clone Mtl 25 apresentando a maior produção de matéria seca. No entanto, não foram observadas diferenças significativas no crescimento radicular entre os dois materiais genéticos.

A variedade Catuaí Amarelo, de modo geral, apresentou melhor desempenho das características relacionadas ao crescimento vegetativo, na presença do $\mathrm{Al}$, quando comparada ao conilon. Resultados semelhantes foram encontrados por diversos autores (Bragança et al.,1985; Passo \& Ruiz, 1995), os quais atribuíram maior tolerância ao $\mathrm{Al}^{3+}$ à variedade Catuaí Amarelo, quando comparada com o conilon. Esses resultados são contrários aos obtidos por Pavan \& Bingham (1982), estudando Coffea canephora e Coffea arabica. 
Quadro 1. Valores médios da produção de matéria seca de parte aérea e raízes, da área e do comprimento radicular dos clones de café conilon (Coffea canephora) e do Catuaí Amarelo (Coffea arabica), considerando a atividade de $\mathrm{Al}^{3+}$ na solução nutritiva

\begin{tabular}{|c|c|c|c|}
\hline \multirow{2}{*}{ Atividade de alumínio } & \multicolumn{2}{|c|}{ Coffea canephora } & \multirow{2}{*}{$\begin{array}{c}\text { Coffea arabica } \\
\text { (Catuaí Amarelo) }\end{array}$} \\
\hline & Mtl 25 & Mtl 27 & \\
\hline$\mu \mathrm{mol} \mathrm{L}{ }^{-1}$ de $\mathrm{Al}^{3+}$ & \multicolumn{3}{|c|}{ Matéria seca de parte aérea ( $\mathrm{g} /$ planta) } \\
\hline 20,68 & 9,13 & 5,61 & 10,58 \\
\hline 50,59 & 10,48 & 6,10 & 10,76 \\
\hline 132,9 & 5,15 & 4,35 & 10,57 \\
\hline 330,4 & 3,46 & 3,24 & 6,00 \\
\hline Médias & 7,06 & 4,83 & 9,48 \\
\hline $\mathrm{CV}(\%)$ & 24,51 & & \\
\hline Conilon vs Catuaí ${ }^{(1)}$ & $7,07 * *$ & & \\
\hline \multirow{2}{*}{ Mtl 25 vs Mtl $27^{(2)}$} & $-2,23^{* *}$ & & \\
\hline & \multicolumn{3}{|c|}{ Matéria seca de raízes (g/planta) } \\
\hline 20,68 & 2,07 & 1,60 & 2,78 \\
\hline 50,59 & 2,88 & 1,15 & 3,02 \\
\hline 132,9 & 1,57 & 1,21 & 3,23 \\
\hline 330,4 & 0,65 & 0,80 & 2,26 \\
\hline Médias & 1,79 & 1,19 & 2,82 \\
\hline $\mathrm{CV}(\%)$ & 35,67 & & \\
\hline Conilon vs Catuaí ${ }^{(1)}$ & $2,66^{* *}$ & & \\
\hline \multirow[t]{2}{*}{ Mtl 25 vs Mtl $27^{(2)}$} & $-0,60^{* *}$ & & \\
\hline & \multicolumn{3}{|c|}{ Área radicular $\left(\mathrm{cm}^{2} /\right.$ planta $)$} \\
\hline 20,68 & 392 & 320 & 584 \\
\hline 50,59 & 527 & 247 & 612 \\
\hline 132,9 & 238 & 220 & 750 \\
\hline 330,4 & 83 & 108 & 401 \\
\hline Médias & 310 & 224 & 587 \\
\hline $\mathrm{CV}(\%)$ & 41,05 & & \\
\hline Conilon vs Catuaí ${ }^{(1)}$ & $639 * *$ & & \\
\hline \multirow[t]{2}{*}{ Mtl 25 vs Mtl $27^{(2)}$} & -86 & & \\
\hline & \multicolumn{3}{|c|}{ Comprimento radicular (cm/planta)) } \\
\hline 20,68 & 6.545 & 5.293 & 8.380 \\
\hline 50,59 & 9.484 & 3.868 & 10.993 \\
\hline 132,9 & 3.344 & 3.531 & 12.385 \\
\hline 330,4 & 1.232 & 1.291 & 5.940 \\
\hline Médias & 5.151 & 3.496 & 9.425 \\
\hline CV (\%) & 48,67 & & \\
\hline Conilon vs Catuaí ${ }^{(1)}$ & $10.202^{* *}$ & & \\
\hline Mtl 25 vs Mtl $27^{(2)}$ & -1.655 & & \\
\hline
\end{tabular}

${ }^{(1)}$ Contraste $(-1-1+2) .{ }^{(2)}$ Contraste $(-1+10) .{ }^{* *}$ : Significativo a $1 \%$ pelo teste F.

Um importante fator que pode ter contribuído para obtenção de resultados diferentes, em relação aos obtidos por Pavan \& Bingham (1982), é a idade das plantas submetidas ao estresse por $\mathrm{Al}^{3+}$, principalmente no tocante à matéria seca inicial. Plantas com maior matéria seca podem ser menos afetadas pelo $\mathrm{Al}^{3+}$, devido à maior disponibilidade de carboidratos $\mathrm{e}$ possibilidade de produção de ácidos orgânicos que complexam $\mathrm{Al}^{3+}$, minimizando os efeitos deste elemento. No presente ensaio, apesar da tentativa de padronização e homogeneização das mudas utilizadas, houve diferença no tipo de propagação para obtenção destas, o que levou a padrões diferenciados de crescimento do sistema radicular e da parte aérea. Assim, as mudas de café da variedade Catuaí Amarelo, propagadas por sementes, apresentavam idade mais 
avançada dos tecidos constituintes da raiz, quando comparadas àquelas dos clones de café conilon, propagados por estaquia, sendo, portanto, menos sensíveis às condições iniciais de toxidez por $\mathrm{Al}$.
A variedade Catuaí Amarelo apresentou, consistentemente, os maiores valores desses nutrientes no tecido vegetal (Quadro 2). A manutenção de teores adequados de $\mathrm{Ca}$ e $\mathrm{P}$ no tecido vegetal pode ter sido

Quadro 2. Teores médios de cálcio e fósforo nas folhas e raízes dos clones de café conilon (Coffea canephora) e do Catuaí Amarelo (Coffea arabica), considerando a atividade de $\mathrm{Al}^{3+}$ na solução nutritiva

\begin{tabular}{|c|c|c|c|}
\hline \multirow[b]{2}{*}{ Atividade de $\mathrm{Al}^{3+}$} & \multicolumn{2}{|c|}{ Coffea canephora } & \multirow{2}{*}{$\begin{array}{l}\text { Coffea arabica } \\
\text { (Catuaí Amarelo) }\end{array}$} \\
\hline & Mtl 25 & Mtl 27 & \\
\hline$\mu \mathrm{mol} \mathrm{L}{ }^{-1}$ de $\mathrm{Al}^{3+}$ & \multicolumn{3}{|c|}{ P nas folhas (dag kg ${ }^{-1}$ ) } \\
\hline 20,68 & 0,28 & 0,26 & 0,49 \\
\hline 50,59 & 0,24 & 0,20 & 0,49 \\
\hline 132,9 & 0,14 & 0,21 & 0,42 \\
\hline 330,4 & 0,18 & 0,08 & 0,22 \\
\hline Médias & 0,21 & 0,18 & 0,41 \\
\hline CV (\%) & 8,66 & & \\
\hline Conilon vs Catuaí ${ }^{(1)}$ & $0,42^{* *}$ & & \\
\hline \multirow[t]{2}{*}{ Mtl 25 vs Mtl $27^{(2)}$} & $-0,024^{*}$ & & \\
\hline & \multicolumn{3}{|c|}{$\mathrm{P}$ nas raízes (dag kg ${ }^{-1}$ ) } \\
\hline 20,68 & 0,47 & 0,44 & 0,74 \\
\hline 50,59 & 0,67 & 0,72 & 0,66 \\
\hline 132,9 & 0,44 & 0,47 & 0,67 \\
\hline 330,4 & 0,48 & 0,41 & 0,51 \\
\hline Médias & 0,51 & 0,51 & 0,65 \\
\hline CV (\%) & 10,95 & & \\
\hline Conilon vs Catuaíi ${ }^{(1)}$ & $0,27^{* *}$ & & \\
\hline \multirow[t]{2}{*}{ Mtl 25 vs Mtl $27^{(2)}$} & $-0,0024$ & & \\
\hline & \multicolumn{3}{|c|}{ Ca nas folhas (dag $\mathrm{kg}^{-1}$ ) } \\
\hline 20,68 & 0,58 & 0,74 & 0,73 \\
\hline 50,59 & 0,67 & 0,76 & 0,97 \\
\hline 132,9 & 0,62 & 0,65 & 0,87 \\
\hline 330,4 & 0,40 & 0,52 & 0,75 \\
\hline Médias & 0,57 & 0,67 & 0,83 \\
\hline $\mathrm{CV}(\%)$ & 12,62 & & \\
\hline Conilon vs Catuaí ${ }^{(1)}$ & $0,42^{* *}$ & & \\
\hline \multirow[t]{2}{*}{ Mtl 25 vs Mtl $27^{(2)}$} & $0,099^{*}$ & & \\
\hline & \multicolumn{3}{|c|}{ Ca nas raízes (dag kg ${ }^{-1}$ ) } \\
\hline 20,68 & 0,31 & 0,25 & 0,47 \\
\hline 50,59 & 0,25 & 0,23 & 0,43 \\
\hline 132,9 & 0,21 & 0,18 & 0,28 \\
\hline 330,4 & 0,18 & 0,14 & 0,32 \\
\hline Médias & 0,24 & 0,20 & 0,38 \\
\hline CV (\%) & 19,07 & & \\
\hline Conilon vs Catuaíi ${ }^{(1)}$ & $0,32^{*}$ & & \\
\hline Mtl 25 vs Mtl $27^{(2)}$ & $-0,037^{\circ}$ & & \\
\hline
\end{tabular}

${ }^{(1)}$ Contraste $(-1-1+2) .{ }^{(2)}$ Contraste $(-1+10) .{ }^{\circ}, * \mathrm{e}^{* *}$ : Significativos a 10,5 e $1 \%$ pelo teste $\mathrm{F}$. 
um importante diferencial no melhor desempenho do crescimento vegetativo, observado na variedade Catuaí Amarelo (Quadro 1).

Theodoro et al. (2003), revisando diferentes autores, observaram que os teores adequados de $\mathrm{P}$ em folhas de cafeeiro (arábica) podem variar de 1,2 a 2,2 $\mathrm{g} \mathrm{kg}^{-1}$, e os teores de Ca, de 7,5 a $15 \mathrm{~g} \mathrm{~kg}^{-1}$. Valarini et al. (2005), estudando 12 cultivares de café arábica, em três diferentes épocas, encontraram valores médios de $\mathrm{P}$ de $1,2 \mathrm{~g} \mathrm{~kg}^{-1}$, variando de 0,9 a $1,5 \mathrm{~g} \mathrm{~kg}^{-1}$. Os teores de médios de Ca foram de 16,3 $\mathrm{g} \mathrm{kg}^{-1}$, variando de 11,4 a $20,8 \mathrm{~g} \mathrm{~kg}^{-1}$.

Os teores de $\mathrm{Al}$ são maiores nas raízes, seguidos por folhas e caules (Quadro 3). A variedade Catuaí Amarelo apresentou menores teores de $\mathrm{Al}$ na parte aérea (folhas e caules) e maiores nas raízes, quando comparada ao conilon.

A complexação de $\mathrm{Al}$ na raiz, evitando seu transporte à parte aérea da planta, pode ser um importante fator para a tolerância de plantas ao $\mathrm{Al}$,

Quadro 3. Teores médios de alumínio nas folhas, caules e raízes dos clones de café conilon (Coffea canephora) e do Catuaí Amarelo (Coffea arabica), considerando a atividade de $\mathrm{Al}^{3+}$ na solução nutritiva

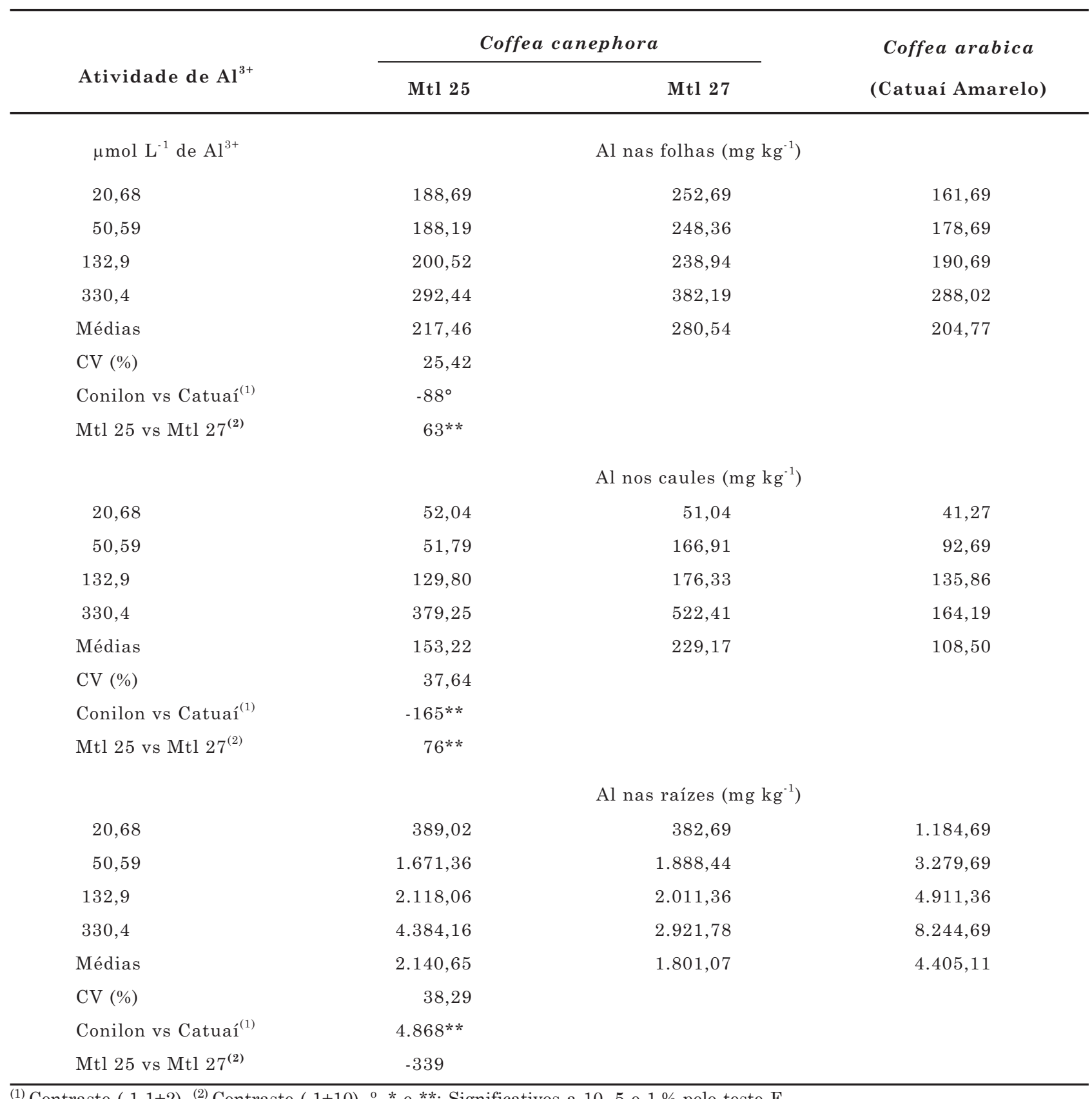

${ }^{(1)}$ Contraste $(-1-1+2) .{ }^{(2)}$ Contraste $(-1+10) .{ }^{\circ},{ }^{*} \mathrm{e}^{* *}$ : Significativos a 10,5 e $1 \%$ pelo teste F. 
pois a permanência do íon no sistema radicular pode evitar efeitos deletérios desse elemento em outros órgãos. Os resultados sugerem mecanismos internos de tolerância ao $\mathrm{Al}$ para a variedade Catuaí Amarelo. Diversos autores relatam, como mecanismos internos de tolerância, a complexação do $\mathrm{Al}$ no citossol por ligantes como ácidos orgânicos e proteínas, a compartimentalização no vacúolo e a existência de enzimas de tolerância ao Al (Rengel \& Reid, 1997; Haug \& Vitorelo, 1997; Kochian \& Jones, 1997).

Foi observado, na variedade Catuaí Amarelo e no clone de conilon Mtl 25, incremento na produção de matéria seca e crescimento radicular em baixas concentrações de atividade de $\mathrm{Al}^{3+}$ na solução nutritiva. $\mathrm{O}$ clone Mtl 27 mostrou-se mais sensível à presença de $\mathrm{Al}$, apresentando resposta negativa do crescimento vegetativo em atividades baixas de $\mathrm{Al}^{3+}$ em solução, exceto para produção de matéria seca de parte aérea.

Estímulos ao crescimento de plantas, quando cultivadas em meios com concentrações baixas e intermediárias de $\mathrm{Al}$, são verificados com freqüência na literatura (Watanabe et al., 1997; Osaki et al., 1997). O efeito de atividades baixas a intermediárias do $\mathrm{Al}^{3+}$ no crescimento da variedade Catuaí Amarelo e do clone Mtl 25, possivelmente, está relacionado à minimização do efeito tóxico do $\mathrm{H}^{+}$devido a um deslocamento eletrostático pelo $\mathrm{Al}^{3+}$ na superfície da membrana plasmática (Kinraide, 2003). O Al tornouse tóxico para as plantas de Catuaí Amarelo (Coffea arabica) a partir da atividade de $76 \mu \mathrm{mol} \mathrm{L} \mathrm{L}^{-1} \mathrm{de}^{3+}$. Para o clone Mtl 25 (Coffea canephora), a toxicidade ocorreu em valores superiores a $35 \mu \mathrm{mol} \mathrm{L}{ }^{-1} \mathrm{de} \mathrm{Al}^{3+}$. O clone Mtl 27, mais sensível, apresentou redução de matéria seca de raiz e do crescimento radicular na presença do $\mathrm{Al}$.

$\mathrm{O}$ aumento da atividade de $\mathrm{Al}^{3+}$ na solução nutritiva reduziu os teores foliares de $\mathrm{P}$ nas duas espécies estudadas. A variedade Catuaí Amarelo apresentou redução nos teores de $\mathrm{P}$ nas raízes, enquanto nos clones de conilon houve aumento dos teores desse nutriente nas raízes, em baixas atividades de $\mathrm{Al}^{3+}$, com posterior redução (Quadro 2).

Os teores de Ca, nas folhas da variedade Catuaí Amarelo e do clone Mtl 25, apresentaram aumento em baixas atividades de $\mathrm{Al}^{3+}$ e posterior redução, com o aumento da atividade do íon em solução. Para o clone Mtl 27 foi observada redução dos teores foliares de Ca com o aumento da atividade de $\mathrm{Al}^{3+}$ na solução nutritiva. Houve redução dos teores de Ca nas raízes das duas espécies estudadas com o aumento da atividade de $\mathrm{Al}^{3+}$. A presença de $\mathrm{Al}$ pode ter reduzido a concentração de Ca no tecido, pelo fato de ambos os íons competirem pelo mesmo sítio do carregador ativo no processo de absorção, ocorrendo inibição competitiva do $\mathrm{Al}$ com o Ca (Malavolta et al., 1997).

A alteração na absorção e no transporte de $\mathrm{P}$ e Ca em plantas expostas ao Al é algo bastante conhecido.
$\mathrm{O}$ Al prejudica a absorção de $\mathrm{P}$ de duas formas: reduz o crescimento do sistema radicular (Foy, 1976) e ligase ao $\mathrm{P}$, tanto na superfície quanto no interior das células, formando compostos de baixa solubilidade (Clarkson, 1966). Dessa forma, ocorre redução na absorção de $\mathrm{P}$, bem como precipitação do nutriente nas raízes, restringindo sua translocação para a parte aérea. Gaume et al. (2001) propuseram que a tolerância ao $\mathrm{Al}$ por plantas de milho estava associada à imobilização do $\mathrm{Al}$ pelo $\mathrm{P}$ nos tecidos da raiz. Os autores observaram maior capacidade de utilização de $\mathrm{P}$ por cultivares mais tolerantes ao Al. Além disso, Vazquez et al. (1999) relataram que plantas de milho tolerantes ao $\mathrm{Al}$ realizam transporte ativo do complexo Al-P da parede celular para o vacúolo. Zhu et al. (2002) demonstraram que variedades de trigo tolerantes ao $\mathrm{Al}$ utilizaram mais eficientemente o P. Zheng et al. (2005) verificaram que a concentração de $\mathrm{Al}$ e $\mathrm{P}$ foi maior em variedades de trigo tolerantes ao $\mathrm{Al}$ e a atribuíram este fato à maior resistência das variedades que acumulavam maior quantidade de $\mathrm{Al}$ e $\mathrm{P}$ nas raízes. Pellet et al. (1996), também estudando variedades de trigo, verificaram que a tolerância destas ao $\mathrm{Al}$ pode envolver mecanismos múltiplos de tolerância, inclusive a exsudação de fosfato pelo ápice radicular, o que pode precipitar o $\mathrm{Al}^{3+}$, restringindo assim sua absorção. Esse comportamento talvez possa explicar os teores de $\mathrm{P}$ observados na parte aérea da variedade Catuaí Amarelo.

Já o aumento da concentração de Ca em solução diminui o efeito tóxico do $\mathrm{Al}$, por reduzir a atividade do $\mathrm{Al}^{3+}$ na superfície externa da membrana plasmática das células da raiz (Silva et al., 2005).

Os resultados demonstram diferenças entre os materiais genéticos de café quanto à toxidez por $\mathrm{Al}$. A comparação entre as duas espécies de café (Coffea canephora e Coffea arabica), em ensaios de campo, torna-se dificultada pela exigência diferenciada de clima e solo, pois os efeitos do $\mathrm{Al}$ seriam sobrepostos por demais fatores, principalmente o clima. No entanto, ensaios que contemplem cada espécie são essenciais na distinção dos materiais genéticos. A confirmação mais aprimorada dessas diferenças e identificação dos materiais genéticos do cafeeiro podem ter implicação na alocação dos clones ou variedades em áreas de produção onde o problema de toxidez por $\mathrm{Al}$ é comum, aumentando a eficiência do sistema produtivo e reduzindo custos de produção. Por outro lado, a utilização de clones ou variedades de café mais sensíveis ao Al implicaria o incremento das doses de calcário e conseqüente neutralização desse elemento.

No quadro 4 são apresentadas as equações de regressão que descrevem o comportamento da variedade Catuaí Amarelo e dos dois clones de conilon, em resposta à atividade de $\mathrm{Al}^{3+}$ na solução nutritiva. 
Quadro 4. Equações de regressão que descrevem a produção de matéria seca da parte aérea e raízes, da área e do comprimento radicular, dos teores de fósforo, cálcio e alumínio, da variedade Catuaí Amarelo e dos clones Mtl 25 e Mtl 27 de café conilon, considerando a atividade de $\mathrm{Al}^{3+}$ em solução nutritiva

\begin{tabular}{|c|c|c|}
\hline Genótipo & Equação de regressão & $\mathbf{R}^{2}$ \\
\hline \multicolumn{3}{|c|}{ Matéria seca de parte aérea } \\
\hline Catuaí Amarelo & $\hat{y}=10,37+0,01134 x-0,0000744 x^{2}$ & 0,999 \\
\hline Mtl 25 & $\hat{y}=10,19 \exp \left\{-1 / 2[\ln (x / 34,86) / 1,349]^{2}\right\}$ & 0,922 \\
\hline Mtl 27 & $\hat{y}=5,94 \exp \left\{-1 / 2[\ln (x / 34,86) / 1,950]^{2}\right\}$ & 0,953 \\
\hline \multicolumn{3}{|c|}{ Matéria seca de raiz } \\
\hline Catuaí Amarelo & $\hat{y}=2,640+0,00835 x-0,0000288 x^{2}$ & 0,997 \\
\hline Mtl 25 & $\hat{y}=2,820 \exp \left\{-1 / 2[\ln (x / 45,323) / 1,068]^{2}\right\}$ & 0,982 \\
\hline Mtl 27 & $\hat{y}=1,526-0,00349 x+0,0000041 x^{2}$ & 0,774 \\
\hline \multicolumn{3}{|c|}{ Área radicular $\left(\mathrm{cm}^{2}\right)$} \\
\hline Catuaí Amarelo & $\hat{y}=505,785+3,1610^{\circ} \mathrm{x}-0,0105^{*} \mathrm{x}^{2}$ & 0,980 \\
\hline Mtl 25 & $\hat{y}=529,99 \exp \left\{-1 / 2[\ln (x / 41,705) / 0,9634]^{2}\right\}$ & 0,988 \\
\hline Mtl 27 & $\hat{y}=1 /(0,00265+0,0000191 x)$ & 0,969 \\
\hline \multicolumn{3}{|c|}{ Comprimento radicular $(\mathrm{cm})$} \\
\hline Catuaí Amarelo & $\hat{y}=-160,665+2379,860^{* *} x^{0,5}-112,432^{*} x$ & 0,999 \\
\hline Mtl 25 & $\hat{y}=9678,52 \exp \left\{-1 / 2[\ln (x / 41,429) / 0,8313]^{2}\right\}$ & 0,981 \\
\hline Mtl 27 & $\hat{\mathrm{y}}=5520,770-0,995^{* *} \mathrm{x}$ & 0,966 \\
\hline \multicolumn{3}{|c|}{ P nas folhas (dag kg ${ }^{-1}$ ) } \\
\hline Catuaí Amarelo & $\hat{y}=0,52840-0,000907^{* * *} x$ & 0,988 \\
\hline Mtl 25 & $\hat{y}=0,317-0,00191^{*} x+0,00000454^{*} x^{2}$ & 0,998 \\
\hline Mtl 27 & $\hat{\mathrm{y}}=1 /\left(2,895+0,0279^{* *} \mathrm{x}\right)$ & 0,910 \\
\hline \multicolumn{3}{|c|}{ P nas raízes (dag kg ${ }^{-1}$ ) } \\
\hline Catuaí Amarelo & $\hat{\mathrm{y}}=1 /\left(1,336+0,0018^{* *} \mathrm{x}\right)$ & 0,911 \\
\hline Mtl 25 & $\hat{y}=0,372+(21,267 / x)-\left(387,11 / x^{2}\right)$ & 0,779 \\
\hline Mtl 27 & $\hat{y}=0,287+(33,398 / x)-\left(604,52 / x^{2}\right)$ & 0,963 \\
\hline \multicolumn{3}{|c|}{ Ca nas folhas (dag $\mathrm{kg}^{-1}$ ) } \\
\hline Catuaí Amarelo & $\hat{y}=0,49540+0,07909^{* *} x^{0,5}-0,003620^{* *} x$ & 0,605 \\
\hline Mtl 25 & $\hat{y}=0,405+0,0546^{\circ} x^{0,5}-0,003029^{*} x$ & 0,972 \\
\hline Mtl 27 & $\hat{\mathrm{y}}=1 /\left(1,264+0,00203^{(1)} \mathrm{x}\right)$ & 0,985 \\
\hline \multicolumn{3}{|c|}{ Ca nas raízes (dag kg ${ }^{-1}$ ) } \\
\hline Catuaí Amarelo & $\hat{y}=0,5356-0,002645^{* *} \mathrm{x}+0,00000603^{* *} \mathrm{x}^{2}$ & 0,983 \\
\hline Mtl 25 & $\hat{\mathrm{y}}=0,183+2,733^{* *} / \mathrm{x}$ & 0,986 \\
\hline Mtl 27 & $\hat{\mathrm{y}}=1 /\left(3,931+0,0101^{* *} \mathrm{x}\right)$ & 0,967 \\
\hline \multicolumn{3}{|c|}{ Al nas folhas $\left(\mathrm{mg} \mathrm{kg}^{-1}\right)$} \\
\hline Catuaí Amarelo & $\hat{\mathrm{y}}=1 /\left(0,00623-0,00000829^{* *} \mathrm{x}\right)$ & 0,981 \\
\hline Mtl 25 & $\hat{\mathrm{y}}=1 /\left(0,00560-0,00000635^{* *} \mathrm{x}\right)$ & 0,963 \\
\hline Mtl 27 & $\hat{\mathrm{y}}=221,361+0,442^{* *} \mathrm{x}$ & 0,825 \\
\hline \multicolumn{3}{|c|}{ Al nos caules $\left(\mathrm{mg} \mathrm{kg}^{-1}\right)$} \\
\hline Catuaí Amarelo & $\hat{y}=158,788-2556,79^{* * *} / x$ & 0,945 \\
\hline Mtl 25 & $\hat{\mathrm{y}}=5,904+1,102^{* *} \mathrm{x}$ & 0,982 \\
\hline Mtl 27 & $\hat{y}=39,079+1,422^{* *} x$ & 0,949 \\
\hline \multicolumn{3}{|c|}{ Al nas raízes $\left(\mathrm{mg} \mathrm{kg}^{-1}\right)$} \\
\hline Catuaí Amarelo & $\hat{y}=-1380,09+640,327^{\circ} x^{0,5}-6,254 x$ & 0,988 \\
\hline Mtl 25 & $\hat{\mathrm{y}}=587,721+11,620^{* *} \mathrm{x}$ & 0,947 \\
\hline Mtl 27 & $\hat{y}=2776,84-49611,3^{* *} / x$ & 0,924 \\
\hline
\end{tabular}

${ }^{\circ},{ }^{*} \mathrm{e}^{* *}$ : Significativos a 10,5 e $1 \%$ pelo teste $\mathrm{F}$. 


\section{CONCLUSÕES}

1. A variedade Catuaí Amarelo apresentou-se menos sensível ao $\mathrm{Al}^{3+}$ do que os clones de conilon; o clone de conilon Mtl 25 é menos sensível ao $\mathrm{Al}^{3+}$ do que o clone Mtl 27.

2. $\mathrm{O}$ aumento da atividade de $\mathrm{Al}^{3+}$ promoveu a redução nos teores de $\mathrm{P}$ e Ca nas folhas e raízes do cafeeiro, especialmente nos clones Mtl 25 e Mtl 27.

3. O acúmulo de $\mathrm{Al}$ no sistema radicular e a restrição do transporte para a parte aérea são importantes fatores na maior tolerância de plantas ao $\mathrm{Al}^{3+}$.

\section{LITERATURA CITADA}

BOLLARD, E.G. Involvement of unusual elements in plant growth and nutrition. In: LAUCHLI, A. \& BRELESK, R.L., eds. Encyclopedia of plant physiology. Berlin, Springer-Verlag, 1983. p.695-744.

BRAGANÇA, J.B.; MATIELLO, J.B. \& FABRIS, E.J. Efeito do alumínio trocável do solo sobre o crescimento inicial do cafeeiro conilon (Coffea canephora) em comparação com o Catuaí (Coffea arabica). In: CONGRESSO BRASILEIRO DE PESQUISAS CAFEEIRAS, 12. Caxambu, 1985. Anais. Rio de Janeiro, SEPRO/DEPET/ DIPRO/IBC, 1985. p.112-114.

CAMBrAiA, J.; GALVANI, F.R.; ESTEVÃO, M.M. \& SANTANNA, R. Effects of aluminum on organic acid, sugar and aminoacid composition of the root system of sorghum (Sorghum bicolor L. Moench). J. Plant Nutr., 6:313-322, 1983.

CLARKSON, D.T. Effect of aluminum on the uptake and on metabolism of phosphorus by barley seedlings. Plant Physiol., 41:165-172, 1966.

CLARKSON, D.T. Interactions between aluminum and phosphorus on root surface and cell wall material. Plant Soil, 27:347-56, 1967.

FOY, C.D. Differential aluminum and manganese tolerances of plant species and varieties in acid soils. Ci. Cult., 28:150155,1976

FOY, C.D. The physiology of plant adaptation to mineral stress. Iowa State J. Res., 57:355-391, 1983.

GAUME, A.; MACHLER, F. \& FROSSARD, E. Aluminum resistance in two cultivars of Zea may L.: Root exudation of organic acids and influence of phosphorous nutrition. Plant Soil, 234:73-81, 2001.

HAUG, A.R. \& VITORELLO, V. Cellular aspects of aluminum toxicity in plants. In: YASUI, M.; STRONG, M.J.; OTA, K. \& VERITY, M.A., eds. Mineral and metal neurotoxicology. Boca Raton, CRC Press, 1997. p.35-41.
HUANG, J.W.; PELLET, D.M.; PAPERNIK, L.A. \& KOCHIAN, L.V. Aluminum interactions with voltage-dependent calcium transport in plasma membrane vesicles isolated from roots of aluminum-sensitive and resistant wheat cultivars. Plant Physiol., 110:561-569, 1996.

JORGE, L.A.C. \& CRESTANA, S. SIARCS 3.0: Novo aplicativo para análise de imagens digitais aplicado a ciência do solo. In: CONGRESSO LATINO-AMERICANO DE CIÊNCIA DO SOLO, 13., Águas de Lindóia. Anais. Campinas, Sociedade Brasileira de Ciência do Solo, 1996. CD-ROM.

KINRAIDE, T.B. Toxicity factors in acidic forest soils: Attempts to evaluate separately the toxic effects of excessive $\mathrm{Al}^{3+}$ and $\mathrm{H}^{+}$and insufficient $\mathrm{Ca}^{2+}$ and $\mathrm{Mg}^{2+}$ upon root elongation. Eur. J. Soil Sci., 54:323-333, 2003.

KOCHIAN, L.V. Cellular mechanisms of aluminum toxicity and resistence in plants. Ann. Rev. Plant Physiol. Plant. Molec., 46:237-260, 1995.

KOCHIAN, L.V.; HOEKENGA, O.A. \& PIÑEROS, M.A. How do crop plants tolerate acid soils? Mechanisms of aluminum tolerance and phosphorous efficiency. Ann. Rev. Plant Biol., 55:459-493, 2004.

KOCHIAN, L.V. \& JONES, D.L. Aluminum toxicity and resistance in plants. In: YOKEL, R.A. \& GOLUB, M.S., eds. Research issues in aluminum toxicity. London, Taylor \& Francis, 1997. p.69-89.

LÓPEZ-BUCIO, L.; NETO JACOBO, M.F.; RAMIREZRODRIGUES, V. \& HERRARA-ESTELLA, L. Organic acids metabolism in plants: From adaptive physiology to transgenic varieties for cultivation in extreme soils. Plant Sci., 160:1-13, 2000.

MA, Q.F.; RENGEL, Z. \& KUO, J. Aluminum toxicity in rye (Secale cereale): Root growth and dynamics of cytoplasmic $\mathrm{Ca}^{2+}$ in intact root tips. Ann. Bot., 89:241-244, 2002.

MALAVOLTA, E.; VITTI, G.C. \& OLIVEIRA, S.A. Avaliação do estado nutricional das plantas: Princípios e aplicações. Piracicaba, Potafos, 1997. 319p.

MATSUMOTO, H. Cell biology of aluminum toxicity and tolerance in higher plants. Int. Rev. Cytol., 200:1-46, 2000.

OSAKI, M.; WATANABE, T. \& TADANO, T. Beneficial effects of aluminum on growth of plants adapted to low pH soils. Soil Sci. Plant Nutr., 43:551-563, 1997.

PASSO, R.R. \& RUIZ, H.A. Tolerância dos cafeeiros conilon e catuaí à toxidez causada pelo alumínio e manganês. $\mathrm{R}$. Ceres, 42:45-52, 1995.

PAVAN, M.A. \& BINGHAM, F.T. Toxidez de alumínio em cafeeiros cultivados em solução nutritiva. Pesq. Agropec. Bras., 17:1293-1302, 1982.

PELLET, D.M.; PAPERNIK, L.A. \& KOCHIAN, L.V. Multiple aluminum-resistance mechanisms in wheat. Roles of root apical phosphate and malate exudation. Plant Physiol., 112:591-597, 1996

RENGEL, Z. \& REID, R.J. Uptake of Al across the plasma membrane of plant cells. Plant Soil, 192:31-35, 1997.

RENGEL, Z. \& ZHANG, W.H. Role of dynamics of intracellular calcium in aluminum-toxicity syndrome. New Phytol., 159:295-314, 2003. 
RYAN, P.R. \& DELHAIZE, E. Function and mechanism of organic anion exudation from plant roots. Ann. Rev. Plant Physiol. Plant Molec. Biol., 52:527-560, 2001.

SILVA, I.R.; SMYTH, T.J.; BARROS, N.F. \& NOVAIS R.F. Physiological aspects of aluminum toxicity and tolerance in plants. In: ALVAREZ V., V.H.; SCHAEFER, C.E.G.R.; BARROS, N.F.; MELLO, J.W.V. \& COSTA, L.M., eds. Tópicos de ciência do solo. Viçosa, MG, Sociedade Brasileira de Ciência do Solo, 2002. v.2. p.277-335.

SILVA, I.R.; FERRUFINO, A.; SANZONOWICZ, C.; SMYTH, T. J.; ISRAEL, D.W. \& CARTER JUNIOR, T.E. Interactions between magnesium, calcium and aluminum on soybean root elongation. R. Bras. Ci. Solo, 29:747-754, 2005.

THEODORO, V.C.A.; CARVALHO, J.G.; CORRÊA, J.B. \& GUIMARÃES, R.J. Avaliação do estado nutricional de agroecossistemas de café orgânico no Estado de Minas Gerais. Ci. Agrotecnol., 27:1222-1230, 2003.

VALARINI, V.; BATAGLIA, O.C. \& FAZUOLI, L.C. Macronutrients in leaves and fruits of dwarf arabica coffee cultivars. Bragantia, 64:661-672, 2005.
VAZQUEZ, M.; POSCHENRIEDER, C.; CORRALES, I. \& BARCELO, J. Changes in apoplastic aluminum during the initial growth response to aluminum by roots of tolerant maize variety. Plant Physiol., 119:435-444, 1999.

WATANABE, T.; OSAKI, M.; YOSHIHARA, T. \& TADANO, T. Aluminum-induced growth stimulation in relation to calcium, magnesium, and silicate nutrition in Melastoma malabathricum L. Soil Sci. Plant Nutr., 43:827-837, 1997.

YANG, J.L.; ZHENG, S.J.; LIN, X.Y.; TANG, C.X. \& ZHOU, G.D. Genotypic differences among plant species in response to aluminum stress. J. Plant Nutr., 28:949-961, 2005.

ZHENG, S.J.; YANG, J.L.; HE, Y.F.; YU, X.H.; ZHANG, L.; YOU, J.F.; SHEN, R.F. \& MATSUMOTO, H. Immobilization of aluminum with phosphorus in roots is associated with high aluminum resistance in buckwheat. Plant Physiol., 138:297-303, 2005.

ZHU, Y.G.; HE, Y.Q.; SMITH, S.E. \& SMITH, F.A. Buckwheat (Fygopyrum esculentum Moench) has high capacity to take up phosphorous (P) from calcium (Ca)-bound source. Plant Soil, 239:1-8, 2002. 